\title{
Algunos comentarios sobre la valoración de la prueba*
}

\author{
Michele Taruffo
}

Al leer las contribuciones de Perfecto Andrés Ibáñez, de Marina Gascón Abellán y de Andrés Bouzat y Alejandro S. Cantaro encuentro con agrado una convergencia sustancial con las tesis de fondo que he expuesto tanto en el artículo que es objeto de esta discusión como, de una manera más amplia y en profundidad, en otros escritos míos $^{42}$.

Me enfrento, por tanto, más que a objeciones o críticas, a una serie de estimulantes reflexiones que me inducen a profundizar, y quizás también a aclarar, algunos aspectos de mi pensamiento. Para hacer esto me parece oportuno, con el fin de evitar repeticiones, no comentar detalladamente aquello que cada uno de los autores ha escrito, sino concentrar la atención sobre los problemas más importantes que han puesto de manifiesto.

1. Una cuestión que destaca en gran medida, especialmente en las consideraciones de Perfecto Andrés Ibáñez y de Marina Gascón Abellán, se refiere a la inmediación del contacto del juez con la prueba y la concepción según la cual el juez se formaría sobre la prueba una "íntima convicción" subjetiva y no susceptible de ser racionalizada que, en cuanto tal, no podría ni ser expresada ni justificada en la motivación de la sentencia.

Mi opinión al respecto coincide sustancialmente con la expresada por mis dos amigos, en el sentido de que esta orientación está radicalmente equivocada y se funda en una serie de errores y equívocos. Trataré aquí de indicar algunos.

Ante todo, queda excluido -en el plano teórico general- que la discrecionalidad de la valoración que el juez realiza acerca de la credibilidad y la eficacia de la prueba coincida necesariamente con un arbitrio subjetivo, irracional e incontrolable. Una tendencia de este tipo está presente en la cultura europea, bajo el influjo de la

\footnotetext{
* Traducción de Daniel González Lagier.
} 
concepción francesa de la intime conviction, entendida como persuasión subjetiva, intuitiva y fundada en reacciones psicológicas e incluso emotivas del juez, y más en general, por la influencia de concepciones psicologistas e irracionalistas de la decisión judicial. Sin embargo, esto no demuestra que esta tendencia esté fundada. De hecho, existen otras concepciones del "libre convencimiento" del juez que no tienen estas implicaciones subjetivistas sino que, por el contrario, van en la dirección de un convencimiento racional que el juez debe formarse sobre la base de la prueba. Este es el caso, por ejemplo, de la freie Beweiswürdigung, orientada hacia la determinación de la verdad de los hechos, de la que habla el $§ 286$ de la Zivilprozessordnung alemana, y el de las distintas concepciones del razonamiento probatorio que utilizan modelos de tipo probabilístico $^{43}$.

En España, las referencias a las reglas de la sana crítica que se encuentran en varias normas de la Ley de Enjuiciamiento Civil del 2000 (véanse, por ejemplo, los artículos 316.2, 348 y 376) no remiten a las reacciones subjetivas del juez sino a la aplicación de "reglas" que deberían fundar un razonamiento cognoscitiva y lógicamente correcto.

Por otra parte, no hay ninguna razón válida para pensar que cuando el juez entra en contacto inmediato con la prueba, por ejemplo, escuchando a un testigo, sea transportado irremediablemente a una dimensión irracional en la que sólo hay espacio para sus reacciones interiores e individuales. Ciertamente, el juez que escucha a un testigo está expuesto a impresiones inmediatas que pueden ser provocadas por el comportamiento del testigo (su modo de expresarse y de moverse, las expresiones del rostro, el tono y las inflexiones de la voz, etc.). Puede también suceder que estas impresiones influyan en el ánimo del juez cuando deba valorar la fiabilidad del testigo y de su declaración. Sin embargo, éste es el aspecto más incierto y peligroso de la valoración de la prueba, del cual el propio juez debe desconfiar asumiendo una actitud de distancia crítica, más que dejarse envolver emocionalmente por él. Por un lado, se encuentra el peligro de que el juez use en la interpretación del comportamiento del

\footnotetext{
${ }^{42}$ Cfr., en particular, La prueba de los hechos, trad. española de Jordi Ferrer Beltrán, Madrid, 2002, y algunos de los ensayos recogidos en el volumen Sui confini. Scritti sulla giustizia civile, Bolonia, 2002.

${ }^{43}$ Para un análisis de estas concepciones, Cfr. mi trabajo La prueba, cit., pág. 190 ss.. Véase también Schum, Evidential Foundations of Probabilistic Reasoning, New York-Chichester-Bribane-TorontoSingapur, 1994.
} 
testigo criterios psicológicos sin fundamento extraídos de un sentido común que, en realidad, está lleno de errores y de prejuicios: el juez que actúa como psicólogo sin una adecuada formación específica, y que usa la psicología barata de los semanarios populares, corre el riesgo de cometer errores burdos y sustanciales. Los estudios sobre la psicología de las declaraciones testificales muestran que se trata de un fenómeno extremadamente complejo y difícil de interpretar, y que la credibilidad del testigo puede ser evaluada sólo teniendo en cuenta una serie de factores relevantes relativos al funcionamiento de la memoria y a las modalidades de reconstrucción de los hechos percibidos por el testigo ${ }^{44}$. Por otro lado, es evidente que si el juez se deja llevar por sus reacciones emocionales a la búsqueda de una "íntima" convicción inefable e inexplicable, no hace otra cosa que convertir su valoración en puro arbitrio.

El necesario distanciamiento crítico debe llevar al juez ${ }^{45}$ a "desprenderse de sí mismo" y a "observarse desde fuera" para seleccionar, entre los inputs que provienen del contacto inmediato con la prueba, aquellos que pueden tener algún valor cognoscitivo sobre la base de criterios intersubjetivamente aceptados o aceptables y posiblemente fundados en el plano del análisis psicológico, separándolos de aquellos que no tienen este valor y que por ello permanecen dentro de la esfera de las reacciones emotivas estrictamente individuales y no tienen ningún significado intersubjetivo. Los primeros inputs son usados como premisas para inferencias que permiten derivar conclusiones racionalmente justificadas acerca de la credibilidad de los testigos y por tanto acerca de la fiabilidad de sus declaraciones. Los inputs del segundo tipo son eliminados y excluidos de la valoración que el juez hace sobre la fiabilidad de la prueba: precisamente porque se trata de reacciones psicológicas del juez que no racionaliza de acuerdo con criterios intersubjetivamente aceptables, no pueden y no deben interferir o influir en esta valoración. No nos gustaría que nuestro médico formulase su diagnóstico fundándose en sus propias reacciones "íntimas" provocadas por la presencia del

\footnotetext{
${ }^{44}$ Cfr. Mazzoni, Si può credere a un testimone? La testimonianza e le trappole della memoria, Bolonia, 2003.

${ }^{45}$ Sobre el "distanciamiento" como actitud típica del juez en la confrontación de la prueba, véase Taruffo, Involvement and Detachment in the Presentation of Evidence, en Prescriptive Formality and Normative Rationality in Modern Legal Systems. Festschr. for Robert S. Summers, ed. por W. Krawietz-N. MacCormick-G.H. von Wright, Berlín, 1994, p. 385 ss. Más en general sobre el concepto de "distanciamiento" en el conocimiento, véase Elias, Coinvolgimento e distacco. Saggi sulla sociologia della conoscenza, tr. it. Bolonia, 1988, p. 89 ss.
} 
paciente, más que en informaciones controladas y objetivamente atendibles de acuerdo con los criterios de la ciencia médica. De la misma manera, no podemos admitir que el juez decida exclusivamente sobre la base, intuitiva e irreflexiva, de sus emociones individuales.

Todo esto equivale a decir que el juez tiene el deber preciso de extraer, de su contacto directo con la prueba, los factores epistémicamente aceptables. A continuación, sobre la base de estos "datos", debe construir inferencias racionales, fundadas sobre reglas o estándares de valoración que deben ser claramente identificables, sobre todo por el propio juez que los usa. En cierto sentido, lo que no puede ser racionalmente elaborado no existe a los efectos de la correcta valoración de la prueba.

Esto remite a otro problema, que aquí no puede ser adecuadamente discutido pero que debe ser mencionado: el de los criterios con los que el juez debe formular las inferencias en las que se articula su razonamiento. Dejando aparte los casos -por lo demás poco frecuentes- en los que es posible servirse de conocimientos científicos, el juez sólo puede remitirse al sentido común, a la cultura media y a los conocimientos fundados sobre la experiencia común.

No obstante, también en este aspecto del razonamiento del juez existen muchas posibilidades de error. De un lado, el juez no puede hacer otra cosa que recurrir a este pseudo-conocimiento. Sin embargo, de otro lado, el sentido común y la experiencia generan informaciones no controladas, nociones sin fundamento y a menudo falsas, pseudo-reglas extraídas en realidad de errores y prejuicios (muchos están todavía convencidos que el Sol gira en torno a la Tierra $)^{46}$. Esto implica que el juez debe someter a control crítico no sólo los inputs que extrae del contacto directo con la prueba, sino también las reglas y los estándares que usa para interpretar estos inputs y para obtener inferencias a partir de ellos. Es más, el discurso puede ser invertido: frente a las impresiones que le llegan del contacto con la prueba, el juez debe encontrar, en el repertorio de background knowledges que le ofrece la cultura media, estándares creíbles e intersubjetivamente aceptables con los que podrá seleccionar e interpretar los elementos cognoscitivos que le proporciona la prueba. Sólo los elementos que tienen sentido de acuerdo con criterios aceptables de conocimiento pueden ser usados como

\footnotetext{
${ }^{46}$ Para una elaboración más amplia de este tema, véase mi ensayo Senso comune, esperienza e sciencia nel ragionamiento del giudice, ahora en Sui confini, cit., p. 121 ss.
} 
base para las inferencias en las que se articula el razonamiento del juez sobre la prueba. Todo el resto, como ya se ha dicho, es tamquam non esset y debe quedar excluido de la decisión del juez sobre los hechos.

2. En el plano de la teoría de la prueba y de la decisión, me ha parecido útil e interesante la identificación, hecha por Marina Gascón Abellán, de la tendencia que es acertadamente designada como cognoscitivismo acrítico. Este es un buen modo de describir la orientación que, por un lado, desvincula la valoración de la prueba de cualquier criterio racionalmente aceptable de conocimiento de los hechos y, por otro lado, paradójicamente, funda la decisión del juez sobre certezas absolutas que serían incontrovertibles porque representarían el resultado de "estados de conciencia" que se forman en las interiores e inescrutables profundidades del ánimo del juez. Se trata, ciertamente, como dice Marina, de una perversión ideológica de la concepción cognoscitiva o epistémica de la prueba, que trata de fundar una pretendida "convicción cierta" sobre una suerte de intuiciones psicológicas (a veces designadas con nombres enfáticos, como "certeza moral") que no tienen nada que ver con lo que usualmente se entiende por conocer un hecho. Sobre este punto me limito a subrayar que desde el punto de vista del método se trata de una concepción que parece tan ambigua (y quizá precisamente por ello tan difundida) como radicalmente equivocada. El error de base es siempre el mismo, y consiste en pensar que el conocimiento de los hechos en el proceso, a diferencia del conocimiento que se obtiene fuera del proceso, en todos los otros campos de la experiencia, no puede ser otro que el resultado de intuiciones irracionales del sujeto que juzga, y que precisamente por esta razón se trata de un conocimiento no criticable y que escapa de cualquier control y de toda verificación o falsación.

3. El tema del conocimiento de los hechos en el proceso me lleva a hacer algunas consideraciones sobre la verdad judicial de los hechos. En relación con esto debo subrayar que yo también soy de la opinión de que el proceso no es un contexto donde se alcancen verdades absolutas e incontrovertibles (como quizá sucede en la teología y en la metafísica), mucho menos si se pretende que estas verdades se funden en estados de conciencia del juez personales e irracionales. Por el contrario, el proceso es un ámbito en el cual, en el mejor de los casos, se obtienen verdades relativas, contextuales, 
aproximadas, aunque derivadas racionalmente de las pruebas que están a disposición en cada caso particular. De esta premisa de carácter muy general se derivan ciertas consecuencias de algún interés.

En primer lugar, resulta bien fundada la tesis recientemente ilustrada por Jordi Ferrer Beltrán ${ }^{47}$ según la cual puede ser "tenido por verdadero" aquello que resulta probado, y en la medida en que resulta probado. En consecuencia, aunque es necesario que la decisión se funde en la mejor aproximación posible a la realidad empírica de los hechos, es inevitable que se trate en todo caso de una aproximación "relativa", en función de la extensión y de la utilidad epistémica de las pruebas disponibles ${ }^{48}$. Esto abre también la posibilidad de que la decisión pueda fundamentarse en una determinación de los hechos no verdadera, o sobre una aproximación no adecuada, a los hechos del caso, en el supuesto de que las normas jurídicas (como las reglas de prueba legal) o la insuficiencia de elementos probatorios (quizá porque se han excluido pruebas relevantes de acuerdo con normas de inadmisibilidad) llevan al juez a adoptar una decisión que no es conforme a la realidad empírica de los hechos ${ }^{49}$.

Todo esto, no obstante, no convierte en válida y aceptable la opinión, muy difundida en la doctrina procesalista de varios países, según la cual existirían al menos dos verdades: una verdad exclusivamente "formal" o "legal" que se alcanza en todo caso en el proceso, y una verdad "empírica", "histórica" o "material" que existiría y podría ser establecida fuera del proceso pero que es inalcanzable por definición para el juez. Esta duplicación de conceptos de verdad es inútil y equivocada ${ }^{50}$, porque no es verdad que en el ámbito del proceso se persiga una verdad cualitativamente distinta de aquella que existiría en cualquier ámbito fuera del proceso. Lo que se persigue en el proceso es la mejor aproximación posible, basada en las pruebas que estén disponibles en el caso concreto, a la verdad "histórica" o "empírica" de los hechos. Esta verdad histórica o empírica, a su vez, no "existe por sí misma" en algún lugar extraño y lejos del proceso. Es, más bien, una suerte de ideal regulativo, un punto de referencia hacia el que se orienta la actividad de quien, en el proceso o fuera del mismo, tiene la función de averiguar los hechos. Por usar una metáfora fácil, puedo no llegar al Polo Sur, pero si

\footnotetext{
${ }^{47}$ Cfr. Prueba y verdad en el Derecho, Madrid-Barcelona, 2002.

${ }^{48}$ Cfr. Ferrer Beltrán, op.cit., p. 37 ss.

${ }^{49}$ Cfr. Ferrer Beltrán, op. cit. p. 41 ss, 83 ss.

${ }^{50}$ En el mismo sentido, v. Ferrer Beltrán, op. cit., p. 68 ss.
} 
quiero ir a la Tierra del Fuego debo saber en qué dirección moverme, si deseo actuar racionalmente y llegar a mi objetivo, y para esto me sirve saber dónde se encuentra el Polo Sur.

El argumento, usado a menudo, de que en el proceso no se puede establecer la verdad "plena" de los hechos debido a los límites legales (esto es: normas que excluyen pruebas relevantes) o prácticos (esto es: límites de tiempo y de recursos) que caracterizan la determinación judicial de los hechos, no prueba en absoluto lo contrario de lo que he dicho, sino que más bien lo confirma. Sólo prueba lo que es obvio y nadie discute, esto es, que el proceso es un contexto de búsqueda y aproximación a la verdad que tiene reglas y límites propios, como sucede en el resto de contextos prácticos en los que se averiguan hechos ${ }^{51}$. En definitiva, este argumento no dice nada distinto de lo que se dice cuando se observa que la verdad (como prueba de los hechos) que se puede conseguir en el proceso es relativa y contextual, y por tanto no puede ser otra que una aproximación mejor o peor a la realidad empírica de los hechos que son determinados.

La concepción que se funda en la relatividad contextual de la verdad judicial pero también en su tendencia a reflejar en alguna medida la realidad empírica de los hechos, tiene la ventaja de permitir la comparación y la valoración crítica de los distintos sistemas procesales desde el punto de vista de su capacidad para facilitar mejores o peores aproximaciones a la realidad por parte del juez: un proceso que contenga, por ejemplo, varias reglas de prueba legal y varias reglas de exclusión de pruebas lógicamente relevantes permite una aproximación a la realidad peor que la que puede haber en un proceso sin pruebas legales y donde todas las pruebas relevantes son admisibles.

Esta concepción de la verdad judicial como aproximación a la realidad de los hechos, variable de acuerdo con los contextos procesales que se tomen en consideración, me da la posibilidad de aclarar mi posición sobre un punto discutido por Andrés Bouzat y Alejandro S. Cantaro. Se trata de la distinción entre decisión "justa", en cuanto fundada en la verdad de los hechos, pero que no sería alcanzable en el proceso, y decisión "justificada", en cuanto fundada en una aproximación relativa y contextual que es la única que puede conseguirse en el proceso. Esta distinción tiene, en

\footnotetext{
${ }^{51}$ Análogamente, v. Ferrer Beltrán, op. cit. p. 45 ss.
} 
mi opinión, el inconveniente de reproducir -aunque de manera metodológicamente más sofisticada- el argumento de la "doble verdad" que he criticado. Por otro lado, en el trabajo donde desarrollé de manera más amplia la tesis de que la veracidad de la determinación de los hechos es una de las condiciones necesarias para la justicia de la decisión ${ }^{52}$, especifiqué que en los casos concretos esta condición (como también las otras: la corrección del proceso y la interpretación adecuada de las normas) puede presentarse con distintos grados o niveles de intensidad, en función del grado de aproximación a la realidad que se produzca en cada caso específico. Concluía mi análisis proponiendo una definición de la justicia de la decisión como un algoritmo o combinación de los grados en los que se dan las tres condiciones necesarias de la decisión justa, y subrayaba que la decisión singular puede ser calificada como "más o menos justa" en función de tal algoritmo o combinación, es decir, en función de los grados en los cuales estén presentes las condiciones de la justicia, y puede ser calificada de injusta cuando una de tales condiciones (o más de una) se presenta en un grado inadecuado $^{53}$. En este planteamiento, que todavía creo convincente, se pueden admitir ideas no relativas de justicia y de verdad, pero sólo para indicar el punto teórico en el cual todo el algoritmo, o su componente referida a la determinación de los hechos, alcanzan los valores teóricamente máximos. La decisión en grado máximo o absolutamente justa sería, por tanto, aquella en la cual todas las condiciones de justicia se presentan en el grado ideal y la decisión "verdadera" sería aquella en la cual los hechos se conocen con certeza absoluta. De esta manera, por otra parte, se define un ideal teórico, que puede servir de referente regulativo o de límite, pero que no es concretamente alcanzable en los procesos del mundo real.

Por otra parte, si se continúa esta perspectiva de análisis, la diferencia entre "grados de justicia" de la decisión y decisión "justificada" aparece sólo como una diferencia en el uso de los términos "justo" y "justificado": el primero se refiere al límite teórico hacia el que tiende a aproximarse la decisión, mientras que el segundo indica la situación práctica en el que se encuentra una decisión relativamente justa.

\footnotetext{
${ }^{52}$ Cfr. Idee per una teoria della decisione giusta, ahora en Sui confini, cit., p. 219 ss.

${ }^{53} \mathrm{~V}$. ibidem, p. 229 ss.
} 
4. Otro punto importante, que merece algunas reflexiones adicionales, es el relativo a la justificación del juicio de hecho y de la valoración de la prueba en la motivación de la sentencia.

En relación con esto, quisiera destacar que las concepciones irracionalistas de la valoración de la prueba, de las que ya he hablado antes, cometen nuevos errores y mantienen nuevamente posiciones insostenibles, al excluir la posibilidad de que la valoración de la prueba se justifique con argumentaciones racionales que el juez tiene el deber de exponer en la sentencia.

Entre las varias posibles, quisiera plantear dos objeciones que en mi opinión son fundamentales y suficientes para privar de toda validez a la concepción mencionada: una objeción tiene carácter teórico y la otra es de naturaleza político-jurídica.

La objeción teórica se refiere a la determinación de la naturaleza y de la función de la motivación de la sentencia. La concepción según la cual el juez no puede motivar su valoración de la prueba porque no puede expresar sus estados emotivos íntimos y profundos se funda en una concepción equivocada de la motivación. Según esta idea, la motivación de la sentencia sería una especie de informe, diario o registro del iter psicológico que el juez ha recorrido para llegar a la decisión sobre los hechos: y dado que este iter no puede dejar de ser misterioso y quedar sepultado en los estratos profundos de la conciencia del juez, entonces éste no podría motivar su propio convencimiento sobre los hechos.

La realidad es que la motivación no es y no puede ser un relato de lo que ha sucedido en la mente o en el alma del juez cuando ha valorado la prueba ${ }^{54}$. Las normas que exigen la motivación de la sentencia no reclaman que el juez se confiese reconstruyendo y expresando cuáles han sido los recorridos de su espíritu. Estas normas, por el contrario, le imponen justificar su decisión, exponiendo las razones en forma de argumentaciones racionalmente válidas e intersubjetivamente "correctas" y aceptables ${ }^{55}$. Para decirlo de manera sintética: los procesos psicológicos del juez, sus reacciones íntimas y sus estados individuales de conciencia no le interesan a nadie: lo que interesa

\footnotetext{
${ }^{54}$ Para la demostración de esta afirmación, véase mi trabajo La motivazione della sentenza civile, Padua, 1975 , p. 118 ss, y más recientemente el ensayo Funzione della prova: la funzione dimostrativa, ahora en Sui confini, cit., p. 323 ss.

55 A propósito de esto véase, en particular, Comanducci, Ragionamento giuridico, en Id. Assaggi di metaetica, Turín, 1992, p. 232 ss.
} 
es que justifique su decisión con buenos argumentos.

Naturalmente, esto no excluye a priori que, entre el razonamiento decisorio -con el que el juez valora la prueba y formula la decisión sobre los hechos- y el razonamiento justificativo -con el que el juez motiva la decisión-, puedan existir correspondencias y analogías. Por un lado, el juez que sabe que debe justificar racionalmente la decisión estará probablemente inclinado a valorar la prueba racionalmente y de acuerdo con criterios objetivos, en lugar de dejarse llevar por su subjetividad incontrolada; por otro lado, el juez que motiva justificando su decisión puede perfectamente hacer uso de criterios, razonamientos e inferencias que ha formulado en el momento en el que trataba de llegar a una decisión. Sin embargo, las dos fases del razonamiento del juez, la decisoria y la justificatoria, son cronológica, estructural y funcionalmente distintas, estando la primera orientada a construir la decisión y la segunda a presentar la decisión como justificada sobre la base de buenos argumentos ${ }^{56}$. Pensar que las dos fases del razonamiento coinciden y que la segunda no es otra cosa que una anotación de la primera, es irremediablemente un error que sólo puede cometer quien no conoce y no quiere conocer la realidad de las decisiones judiciales. De esto se sigue que cualquiera que sea el modo como el juez valore la prueba y formule la decisión sobre los hechos, no puede excluirse en absoluto que pueda $\mathrm{y}$, por tanto, deba presentar argumentos racionales para sostener y justificar las decisiones a las que ha llegado sobre la base de la prueba. Llevando al límite al argumento: incluso en el caso de que se admitiese (lo que es necesario excluir por las razones ya expuestas) que el acto de la valoración de la prueba sea un acto irracional, esto no le impediría al juez encontrar argumentos racionalmente aceptables para fundar ex post su decisión; como ya he dicho, a nadie se le reclama que exponga en público sus sensaciones y emociones; lo que se le reclama es otra cosa, esto es, que presente argumentos que legitimen -por decirlo así- hacia fuera la decisión que ha tomado.

Ahora podemos pasar a la objeción jurídico-política que puede dirigirse a la tesis en discusión. La objeción se basa en la función de la motivación y en las razones por las que ésta está prevista como obligatoria, a veces incluso en normas constitucionales. Esta función presenta dos aspectos principales que es útil distinguir. Por un lado, se puede

\footnotetext{
${ }^{56}$ Para un análisis más amplio de estas diferencias, véase mi trabajo La motivazione, cit. p. 222 ss.
} 
hablar de función endoprocesal para aludir al hecho de que la motivación sirve, dentro del proceso, para facilitar a las partes la impugnación de la sentencia y hacer posible que el juez superior pueda ejercer el control de las razones de la decisión dictada por el juez inferior. Por otro lado, se puede hablar de función extraprocesal de la motivación para indicar que ésta sirve para hacer posible, al menos potencialmente, un control externo de las razones que el juez expresa como fundamento de su decisión ${ }^{57}$. Por control externo se entiende el control que debe ser posible por parte de la opinión pública y del ambiente social en la que se inserta la decisión: en este sentido, la obligación de motivar cumple un papel de garantía fundamental de la corrección de la manera como el juez ejerce el poder decisorio del que dispone. Sea en el plano histórico, sea en el de las garantías fundamentales del proceso, la obligación de motivar está directamente conectada con la concepción democrática del poder: es democrático el poder "que se justifica", esto es, que permite a los ciudadanos valorar si ha sido usado de modo correcto y compartible; es absoluto y antidemocrático el poder "que no se justifica", esto es, que no da razón de las decisiones propias y que por tanto escapa de cualquier control social. El juez que no justifica sus propias decisiones en la motivación de la sentencia ejercita el poder del que dispone de manera arbitraria y sustancialmente antidemocrática y viola una de las garantías fundamentales del proceso, precisamente en la medida en que se sustrae al control externo de las razones por las que ha usado su poder de un modo determinado. El juez que ejercita democráticamente el poder del que dispone justifica sus propias elecciones exponiendo, en la motivación de la sentencia, las razones por las que ha adoptado esa decisión, y hace así posible el control crítico difuso sobre la manera como ha ejercitado su poder. La función extraprocesal de la motivación representa un aspecto muy importante de lo que Jerzy Wróblewski ha llamado felizmente ideología legal-racional de la decisión judicial, que caracteriza a los sistemas más evolucionados de administración de la justicia ${ }^{58}$ : es claro, en efecto, que a través de la motivación el juez demuestra la solidez, la legitimidad y la racionalidad de las decisiones.

Estas consideraciones son adecuadas para todos los aspectos de la decisión

\footnotetext{
${ }^{57}$ Sobre esta distinción, ya ampliamente aceptada, véase también mi trabajo La motivazione, cit., p. 370 ss.

${ }^{58}$ Cfr. Wróblewski, "Sentido" y "Hecho" en el derecho, trad. española, San Sebastián, 1988, p. 61 ss.
} 
judicial, pero lo son de un modo particular en lo que se refiere al juicio sobre los hechos y la valoración de la prueba. De hecho, así como no puede hablarse de decisión justa (o justificada) si falta una determinación verdadera de los hechos de la causa, del mismo modo no se puede hablar de una decisión motivada si el juez no indica específicamente, y mediante argumentos racionalmente articulados, las bases cognoscitivas, los criterios de valoración y las inferencias que justifican su valoración de la prueba y el juicio final que deriva de esta valoración. La falta de una adecuada motivación de estos aspectos esenciales de la decisión no implica solamente un ejercicio arbitrario del poder por parte del juez, sino que implica también la violación de una de las garantías fundamentales de la administración de la justicia.

5. Muchos aspectos de las contribuciones a esta discusión se refieren en concreto a problemas de prueba y decisión en el proceso penal, y esto exige, me parece, algunas consideraciones adicionales. Por una parte, en efecto, es verdad que muchos de los discursos que se hacen a propósito de la valoración de la prueba y del juicio sobre los hechos valen tanto para el proceso civil como para el penal y cualquier otro tipo de proceso. Sin embargo, por otra parte, deben tenerse en cuenta algunas características específicas que distinguen al proceso penal del civil.

Uno de estos factores específicos es el desequilibrio sistemático que existe entre las partes del proceso penal, y que condiciona la decisión en función de la presunción de inocencia del acusado. Se trata, como es sabido, de una regla de juicio de acuerdo con la cual el juez debe resolver pro reo la eventual duda que subsista cuando todas las pruebas hayan sido tenidas en cuenta, lo que introduce una distorsión estructural en el criterio de decisión. Naturalmente, al hablar de "desequilibrio" y "distorsión" no quiero sugerir una valoración negativa sobre la presunción de inocencia: pretendo decir que sólo en el proceso penal existe una presunción sistemáticamente favorable a una de las partes, mientras no existe nada igual en el proceso civil, donde las partes se sitúan sistemáticamente en el mismo nivel, y la eventualidad de la ausencia o insuficiente prueba de un hecho se resuelve -de acuerdo con las reglas generales sobre la carga de la prueba- con la derrota de la parte que había alegado ese hecho. De aquí se siguen algunas consecuencias.

Por ejemplo, se suele decir, aunque es un problema muy complejo en el que se 
mezclan distintas concepciones de manera no siempre clara, que en los dos tipos de proceso deberían aplicarse distintos estándares de prueba: en el proceso civil se debería aplicar (como a menudo sucede) el estándar de la probabilidad prevaleciente, de acuerdo con el cual se da por probada la versión de los hechos que aparece como "más probable" a la luz de las pruebas obtenidas, mientras que en el proceso penal debería aplicarse el estándar de la prueba más allá de toda duda razonable, según la cual sólo en el caso de que la prueba haya ofrecido la "certeza" sobre los hechos podría ser condenado el acusado. Estos aspectos no pueden ser discutidos aquí con la amplitud que merecerían; sin embargo conviene señalar que se insertan en el discurso relativo a la prueba de los hechos y a la valoración de la prueba, y que son relevantes al menos en el sentido de hacer necesarias varias distinciones de acuerdo con el tipo de proceso al que se haga referencia. Mi impresión es que el problema de la presunción de inocencia no se formula correctamente cuando se invoca esa presunción para decir que en el proceso penal debe alcanzarse la certeza mientras que en el civil basta la probabilidad, porque en este caso se mezclan los criterios con los que debe adoptarse la decisión en el caso de ausencia de prueba con las modalidades con la que se lleva a cabo la prueba de los hechos; pero la cuestión es muy compleja y por ello me limito a dejarla apuntada, quizá para una futura discusión.

Una característica que en los sistemas más evolucionados es típica del proceso penal, pero tiene también diversas manifestaciones en el proceso civil, se refiere a la distribución de los poderes procesales -en particular de los que se refieren a la adquisición de las pruebas- entre las partes y el juez. Por un lado, en realidad, cuando se habla de las garantías fundamentales de las partes, como el derecho a la defensa y el principio de contradicción, se dice acertadamente que éstas deben ser respetadas íntegramente en los dos tipos de proceso. Por otro lado, sin embargo, la implementación de estas garantías termina produciendo consecuencias distintas en los diversos tipos de proceso. Así, en el ámbito del proceso civil se tiende a pensar que la implementación completa de las garantías de la defensa no excluye en absoluto la posibilidad de atribuir al juez poderes incluso tan amplios como el de disponer de oficio la práctica de pruebas no solicitada por las partes, cuando sea necesario para comprobar la verdad de los hechos. En los procesos civiles modernos hay ejemplos interesantes de esto: el modelo en ciertos aspectos más coherente es el del code de procédure civile francés, cuyo 
artículo 10 prevé un poder general del juez de disponer de oficio la práctica de pruebas no aportadas por las partes, mientras que el artículo 16 prevé la aplicación del principio de contradicción entre las partes en todos los aspectos relevantes de la decisión. Por otra parte, en muchos otros ordenamientos, como por ejemplo en Italia, Alemania e incluso Estados Unidos, se atribuye al juez diversos poderes autónomos de iniciativa instructoria. Existe, por tanto, una tendencia difusa a pensar que un papel activo del juez en la adquisición de la prueba es esencial para la averiguación de la verdad de los hechos en el proceso civil ${ }^{59}$. Las cosas son distintas en el ámbito del proceso penal, donde por muchas razones de carácter histórico y sistemático se tiende a sostener que las garantías fundamentales del proceso sólo pueden ser efectivas con la adopción de un modelo de proceso íntegramente acusatorio, en el que el juez no tenga poderes de iniciativa autónoma en materia de práctica de la prueba, y por tanto toda la actividad probatoria queda remitida exclusivamente a las partes. Obviamente, no tengo ninguna intención de discutir o criticar esta tendencia, que es ampliamente dominante en los procesos penales modernos. Observo sólo que de este modo las líneas evolutivas del proceso penal tienden a divergir profundamente de las líneas evolutivas del proceso civil, al menos en lo que se refiere a la determinación de los sujetos a los que corresponde realizar la adquisición y presentación de las pruebas. Y observo, además, que, quizá por la presencia de la presunción de inocencia, el proceso penal tiende a verse no tanto como un procedimiento orientado al descubrimiento y determinación de la verdad de los hechos sobre la base de todas las pruebas disponibles, sino como un procedimiento orientado a hacer posible para el acusado el desarrollo de todas sus defensas y a verificar si las pruebas presentadas por la acusación son o no suficientes para vencer la presunción de inocencia. Con toda probabilidad, existen óptimas razones para configurar de este modo el proceso penal y no quiero ponerlo en discusión. Sólo señalo que -casi paradójicamente y con una inversión de los términos respecto a lo que sucedía en el pasado-, hoy se tiende a considerar que el proceso civil se orienta a la búsqueda de la verdad, mientras que no se puede decir lo mismo del moderno proceso

\footnotetext{
${ }^{59}$ Prefiero hablar de papel "activo" más que de papel "inquisitivo" del juez civil para evitar los muchos equívocos que el uso de este término provoca a menudo. Sobre la inadecuación de la contraposición usual entre procesos adversarial y procesos inquisitorial, y sobre la oportunidad de no usarla más en la discusión sobre modelos procesales, véase mi trabajo El proceso civile di civil law e di common law: aspetti fondamentali, ahora en Sui confini, cit. p. 73 ss. Véase también Damaška, The Faces of Justice and State Authority. A Comparative Approach to the Legal Process, New Haven-London, 1986, p. 3 ss.
} 
penal.

Además, mientras estas diferencias estructurales deben ser tenidas en cuenta cuando se discute acerca de las funciones del juez, creo que hay muchos aspectos bajo los cuales no hay diferencias relevantes entre el juez civil y el penal. Si se piensa en el momento de la valoración de la prueba y de la decisión sobre los hechos, y si se pone entre paréntesis la cuestión de quién (las partes o el juez) ha propuesto la prueba, se puede pensar que un juez penal que adopte una ideología legal-racional de la decisión formulará su valoración de manera sustancialmente similar a la del juez civil que adopte la misma ideología. Si esto es así, no hay obstáculos para reconocer que también el juez penal, al igual que el juez civil, puede y debe valorar la prueba de manera racional y, por consiguiente, puede y debe justificar su propia decisión sobre los hechos exponiendo "buenas" razones en la motivación de la sentencia.

En cuanto a las modalidades de la decisión sobre los hechos y de la correspondiente motivación, por tanto, puede no haber diferencias estructurales: también un juez que durante el curso del proceso haya desempeñado un papel de "árbitro pasivo" del conflicto que ha surgido entre las partes puede decidir correctamente la causa valorando la prueba de manera racional y justificando adecuadamente la decisión. Esto depende -insisto- de la ideología que el juez tiene respecto de su propio rol, más que del reparto de poderes probatorios entre las partes y el juez. No obstante, este reparto, y por ello también la cuestión de si nos encontramos en un modelo de proceso (civil o penal) completamente dispositivo o acusatorio, en lugar de en un modelo de proceso (civil o penal) en el que el juez está dotado de poderes de instrucción autónomos, tiene relevancia desde otro punto de vista. Como he sugerido antes, en los procesos civiles modernos existe una amplia tendencia (aunque no seguida en algunos sistemas, como en España) a atribuir al juez poderes para ordenar la práctica de pruebas no propuestas por las partes, y normalmente se justifica esta atribución de poderes en el interés de averiguar la verdad y en la imposibilidad de contar exclusivamente con la iniciativa de las partes para la aportación de todas las pruebas que pueden ser necesarias para ello. En definitiva, se asume que el ejercicio de los poderes de oficio del juez es útil para complementar la iniciativa de las partes cuando ésta no es suficiente para aportar todas estas pruebas: entra en juego -al menos de manera tendencial- una especie de criterio de plenitud del material probatorio, de acuerdo con el 
cual todas las pruebas relevantes deben ser adquiridas, a iniciativa de parte o, si es necesario, a iniciativa del juez. Por el contrario, en los sistemas en los cuales el juez no tiene poderes de instrucción autónomos y la aportación de las pruebas es obra exclusivamente de las partes, se corre el riesgo de que las pruebas presentadas por las partes no sean todas aquellas que serían útiles para la determinación de la verdad de los hechos. En un sistema orientado a la búsqueda de la verdad, un riesgo de este tipo ha de valorarse negativamente, y en realidad se recurre al remedio proporcionado por los poderes de instrucción del juez.

En un sistema que no esté orientado a la búsqueda de la verdad, por el contrario, no se considera negativamente el riesgo de que pruebas relevantes o decisivas para la determinación de la verdad queden fuera del proceso, porque por cualquier razón la actividad probatoria de las partes no haya sido la adecuada. Puede darse incluso que la eventual incompletitud de la adquisición de las pruebas se valore positivamente, si la parte que no se arriesga a presentar prueba suficiente es la acusación en un proceso penal: la ineficiencia probatoria de la acusación juega, de hecho, a favor del acusado, que será absuelto con mayor facilidad cuanto más escasa sea la actividad probatoria de la acusación (aunque esto, naturalmente, no juega a favor de la determinación de la verdad). Esta, me parece, es la perspectiva desde la que Andrés Bouzat y Alejandro S. Cantaro manifiestan su opinión contraria a la atribución de poderes autónomos de instrucción al juez penal, en la medida en que estos poderes podrían ser usados a favor de la acusación, y por tanto en sentido contrario a la presunción de inocencia. Es una posición perfectamente respetable, aunque se podría observar que -dado que en el proceso penal también el acusado tiene un fuerte interés en probar su inocencia y no permanece inactivo viendo si la acusación alcanza a probar su culpabilidad- los poderes del juez también podrían usarse a favor del acusado y no sólo a favor de la acusación, así como en el proceso civil pueden ser usados indiferentemente a favor de una u otra parte. A propósito de esto observo solamente que se trata de una posición perfectamente respetable, y también compartible, en el nivel de las elecciones de política del Derecho, puesto que es perfectamente comprensible la preferencia por un sistema que admite el riesgo de la falta de plenitud del material probatorio, si se presupone que este riesgo opera en una única dirección, esto es, a favor del acusado. Pero esto, sin embargo, no impide que en el proceso penal íntegramente acusatorio se pueda tener una composición 
incompleta (respecto a la que vendría representada por todas las pruebas relevantes) de la base probatoria del juicio de hecho, y por tanto distinta de la que puede haber en un proceso civil caracterizado por una función activa del juez y orientado a que todas las pruebas relevantes sean aportadas al juicio. 